\title{
Developing the New Qualitative Measurement Indicator and its Application in Scientific Papers
}

\author{
Yong-Jeong Kim, Dong Hoon Oh \\ Korea Institute of S\&T Evaluation and Planning (KISTEP), Seoul, Korea
}

\begin{abstract}
This article introduces a new qualitative measurement indicator, the Relatively Rank-normalized Impact Factor $\left(R^{2} n I F\right)$ that can overcome drawbacks of the existing qualitative indicators (Journal IF, normalized IF). The qualities of SCI papers in G7 and BRICs are investigated using this indicator. The result of the $R^{2} n I F$ analysis of those countries shows that the US produces not only the largest quantity but also the best quality of SCI papers. The US is the research leader in both "basic science" and "bio-science". The UK also shows high quality in almost all areas, even superior to the US in some research areas such as pharmacology and toxicology, molecular biology and genetics, etc.. Overall the qualitative status of scientific papers in all BRIC countries are not yet up to par, although they have achieved a quantitative expansion of publications. However in "engineering" China is the second only to the US in journal quality.

Keywords: scientific paper, qualitative measurement indicator, Relatively Rank-normalized Impact Factor $\left(R^{2} n I F\right)$, Journal Impact Factor, G7, BRIC
\end{abstract}

\section{Introduction}

The objective measurement of research performance is an important topic in the evaluation and establishment of S\&T policies. The quality of scientific papers is in general the most fundamental means of measuring research performance. However, measuring research performance is not as easy as it appears, and its improper use can be quite misleading.

Certainly, the actual number of citations would provide a better performance indicator. However, this is not ordinarily possible for recently published scientific papers because the time lag of citations makes citation scores almost useless in the evaluation process. In the past, the Journal Impact Factor (IF) has been most commonly used measure of research performance. When using for evaluation purposes, many scholars generally ignore the differences between Journal IF values in different subject categories. For instance, the small number of academic journals in the field of "mathematics" can lead to a low overall Journal IF, while the large number of journals in "bio-science" can lead to a high one. Uninformed scholars still use the Journal IF to compare "bio-science” with "mathematics”. Such inappropriate comparisons of Journal IF between disciplines are irresponsible.

Yong-Jeong Kim, Research Fellow, Office of National R\&D Evaluation and Analysis, Korea Institute of S\&T Evaluation and Planning (KISTEP).

Dong Hoon Oh, Senior Research Fellow, Office of S\&T Policy and Planning, Korea Institute of S\&T Evaluation and Planning (KISTEP).

Correspondence concerning this article should be addressed to Yong-Jeong Kim, Korea Institute of S\&T Evaluation and Planning (KISTEP), Seoul, Korea. E-mail: yongjkim@kistep.re.kr. 
In order to overcome the difficulties of comparing Journal IF across different specialties, Sen (1992) and Marshakova-Shaikevich (1996) suggested using a normalized IF. However, these normalizations are not completely satisfactory, as they use either the maximal Journal IF or a few of the highest Journal IFs in each academic field. In 2004, Pudovkin and Garfield suggested a rank-normalized Impact Factor (rnIF), which is similar to the percentile ranking of Journal IF. However, as a research performance measure the rnIF indicator still fails to take into account the variations between both different academic fields and different countries. This highly complicates comparative analyses and the definition of widely applicable standards of acceptable performance.

This article attempts to develop a new qualitative measurement indicator, the Relatively Rank-normalized Impact Factor $\left(R^{2} n I F\right)$ that can overcome the drawbacks of the existing qualitative measurement indicators such as Journal IF and normalized IF. Furthermore, the qualities of SCI papers in G7 and BRIC countries are investigated with the help of this $R^{2} n I F$ indicator.

\section{Literature Review}

Recently a hot issue has been the use of the Journal Impact Factor (IF) for the evaluation of individual scientists, laboratories, institutions, and R\&D programs. It is not uncommon in some countries to find that grant application reviews generally use the SCI Impact Factor (IF) to evaluate their applicants' scientific papers. Numerous criticisms have been made of the use of SCI IF, including not only its improper use but also the fundamental validity of the SCI IF itself.

It was Sen (1992) who first attempted to correct for the deviation of Journal IF between research areas. Sen suggested the following normalization: $S n I F_{j}=I F_{j} / \operatorname{maxIF} \times 10$, where $I F_{j}$ is the SCI IF for a journal $j$ and maxIF is the maximal IF value for the Journal Citation Reports (JCR) category to which a journal $j$ belongs. Sen's normalized IF was used for the analysis of Indian and Chinese laser physics journals by Garg and Padhi (1999). Also Marshakova-Shaikevich (1996) suggested the following indicator: $M n I F_{j}=I F_{j} /$ av5maxIF $\times 100$, where av5maxIF is the weighted average of the top five SCI IF values in the JCR category to which a journal $j$ belongs. The weighting uses the number of papers in each of the top five journals over a two year period. Marshakova-Shaikevich et al. conducted a bibliometric evaluation of Russian science journals with the help of the MnIF indicator in 2003. However, these measurement indicators (SnIF and MnIF) are not practically applicable due to their large biases between research fields, as criticized by Pudovkin and Garfield (2004).

Pudovkin and Garfield (2004) suggested the rank-normalized Impact Factor ( $r n I F)$. The rnIF values are the percentile rankings for the whole set of journals in JCR categories as shown in equation (1):

$$
r n I F_{j}=\frac{\left(N-R_{j}+1\right)}{N}
$$

The $r n I F_{j}$ is the "rank-normalized Impact Factor" of an academic journal $j, N$ is the number of journals in its category, and $R_{j}$ is the SCI IF ranking within the category of a particular journal $j$.

In order to calculate the $r n I F_{j}$ journals were always displayed in descending order. For instance, the journal "Acta Mathematica” was the 10th from the top in the JCR category for mathematics. This category contained 186 journals, thus the rnIF value of “Acta Mathematics” is $(186-10+1) / 186=0.952$. When $r n I F$ has the value of $x(=0.952)$, it means that $(1-x) \times 100 \%(=4.8 \%)$ of academic journals in the area of mathematics have a higher SCI IF than this journal.

However, a weakness of the the $r n I F$ is that its lowest value is dependent on the number of academic 
journals in the JCR Category. For example, while the lowest $r n I F$ value in JCR category "A" with 10 academic journals is 0.1 , the lowest $r n I F$ value in JCR category " $\mathrm{B}$ " with 100 journals is 0.01 , and the lowest $r n I F$ value in "C" with 1,000 journals is 0.001 . Heo et al. (2008) suggested a modified rank-normalized Impact Factor ( $m r n I F$ ) to overcome this weakness by modifying the $r n I F$ to have a minimum value of 0 and a maximum value of 100 using equation (2):

$$
m r n I F_{j}=100 \times \frac{\left(N \times r n I F_{j}+1\right)}{(N-1)}
$$

The $m r n I F_{j}$ is the "modified rank-normalized Impact Factor" of an academic journal $j, N$ is total number of journals within the JCR category to which a journal $j$ belongs, and $r n I F_{j}$ is the rank-normalized Impact Factor of an academic journal $j$.

However, because the mrnIF does not take into account the different distributions of papers across their journals in different JCR categories, it cannot truly reflect the qualitative status of journals. Table 1 shows well this limitation of comparative analysis using mrnIF indicator (including $r n I F$ ). Assume that there are 1,000 journals each in the fields of "microbiology" and "mathematics" in which two journals, "B" and "b", are ranked second place according to Journal IF. Both journals have the same mrnIF, $100 \times(5 \times 0.8-1) /(5-1)=75$, indicating that the two journals ("B" and "b") have identical journal quality. However this is not truly so since the mrnIF has not taken into account the fields' differing distributions of papers across their journals. While the top-ranked journal in "microbiology", “A”, has 500 papers, that in "mathematics”, "a”, has only 100 papers. Thus, although journal "b" should clearly have a higher qualitative status than journal "B", this is not reflected in either the mrnIF or rnIF at all.

Table 1

Limitation of Comparative Analysis Using the Rank-Normalized Impact Factor

\begin{tabular}{|c|c|c|c|c|c|c|c|c|}
\hline \multirow{2}{*}{ Ranking } & \multicolumn{4}{|c|}{ Microbiology } & \multicolumn{4}{|c|}{ Mathematics } \\
\hline & Journal & Number of papers & $r n I F$ & $m r n I F$ & Journal & Number of papers & $r n I F$ & mrnIF \\
\hline 1 & A & 500 & 1.0 & 100 & a & 100 & 1.0 & 100 \\
\hline 2 & B & 100 & 0.8 & 75 & $\mathrm{~b}$ & 200 & 0.8 & 75 \\
\hline 3 & $\mathrm{C}$ & 200 & 0.6 & 50 & c & 200 & 0.6 & 50 \\
\hline 4 & $\mathrm{D}$ & 100 & 0.4 & 25 & d & 200 & 0.4 & 25 \\
\hline 5 & $\mathrm{E}$ & 100 & 0.2 & 0 & e & 300 & 0.2 & 0 \\
\hline
\end{tabular}

New Qualitative Measurement Indicator: Relatively Rank-Normalized Impact Factor $\left(R^{2} n I F\right)$

This article attempts to develop a new qualitative measurement indicator, the Relatively Rank-normalized Impact Factor $\left(R^{2} n I F\right)$, by modifying the existing rank-normalized Impact Factors ( $\left.r n I F, m r n I F\right)$ so that it can be used to compare the qualitative status of journals between both research areas and countries. We first categorized journals with 22 National Science Indicators (NSI) standard fields on the basis of the JCR 2009 as shown in Table 2, and then calculated both the rnIF and $m r n I F$ values of all journals by equations (1) and (2). Next we calculated the global average of the mrnIF for each NSI standard field. We only needed the total number of papers in each journal to calculate the global average mrnIF for each field. We can get such information using the Science Citation Index Expanded (SCIE) Database. For instance, $m r n I F_{\text {global average }}$ of "mathematics" in year 2009 can be calculated by equation (3). 


$$
m r n I F_{\text {mathematics, }} 2009 \text { global average }=\frac{\sum_{i=1}^{n}\left(m r n I F_{i} \times N_{i}\right)}{\sum_{i=1}^{n} N_{i}}
$$

$N_{i}$ is the number of papers in the $i$ th journal in "mathematics" in 2009, and $m r n I F_{i}$ is $m r n I F$ of the $i$ th journal in "mathematics".

Table 2 shows a global average $m r n I F$ value for each NSI standard field.

Table 2

Global Average of mrnIF in Each NSI Standard Field

\begin{tabular}{|c|c|c|c|}
\hline \multirow{2}{*}{ Field } & \multicolumn{3}{|c|}{$m r n I F_{\text {global average }}$} \\
\hline & 2007 & 2008 & 2009 \\
\hline Agricultural Science & 74.9 & 75.6 & 76.6 \\
\hline Biology \& Biochemistry & 65.6 & 65.4 & 65.3 \\
\hline Chemistry & 67.5 & 68.0 & 69.0 \\
\hline Clinical Medicine & 69.5 & 69.1 & 69.1 \\
\hline Computer Science & 66.0 & 65.9 & 66.6 \\
\hline Economics \& Business & 71.5 & 75.2 & 71.8 \\
\hline Engineering & 71.8 & 71.4 & 72.5 \\
\hline Environment \& Ecology & 70.6 & 70.1 & 72.5 \\
\hline Geoscience & 70.5 & 72.1 & 72.6 \\
\hline Immunology & 63.8 & 63.4 & 62.1 \\
\hline Materials Science & 74.8 & 76.0 & 76.4 \\
\hline Mathematics & 65.9 & 63.8 & 65.4 \\
\hline Microbiology & 88.2 & 88.2 & 88 \\
\hline Molecular Biology \& Genetics & 63.4 & 63.5 & 63.5 \\
\hline Multidisciplinary & 85.9 & 85.1 & 87.2 \\
\hline Neuroscience \& Behavior & 66.4 & 65.9 & 66.2 \\
\hline Pharmacology \& Toxicology & 63 & 62.4 & 63.1 \\
\hline Physics & 67.4 & 67.7 & 67.9 \\
\hline Plant \& Animal Science & 70.4 & 70.9 & 71.2 \\
\hline Psychiatry \& Psychology & 69.4 & 68.6 & 68.6 \\
\hline Social Sciences & 73.3 & 72.7 & 75 \\
\hline Space Science & 75.7 & 71.8 & 75 \\
\hline
\end{tabular}

Finally, we calculated the Relatively Rank-normalized Impact Factor $\left(R^{2} n I F_{j}\right)$ of each paper by dividing the $m r n I F_{j}$ of the journal $j$ by $m r n I F_{\text {global average }}$ of the NSI field to which the journal $j$ belongs as shown in equation (4).

$$
R^{2} n I F_{j}=\frac{m r n I F_{j}}{m r n I F_{\text {global averge of the same field }}}
$$

$R^{2} n I F_{j}$ is the Relatively Rank-normalized Impact Factor of journal $j, m r n I F_{j}$ is modified rank-normalized Impact Factor of journal $j$, and $m r n I F_{\text {global average of the same field }}$ is the global average value of $m r n I F$ of the research field to which journal $j$ belongs.

For example, if the mrnIF value of a paper published in the journal, "Human Genetics", is 79.658, and the $m r n I F_{\text {global average }}$ of the research area, "molecular biology \& genetics", where "Human Genetics" belongs is 63.529, and then the $R^{2} n I F$ value of a paper published in "Human Genetics" is 79.658 / $63.529=1.254$. Table 3 presents further examples of $R^{2} n I F_{j}$ calculations from the fields of "mathematics" and "molecular biology \& genetics”. 


\section{Measuring the Quality of Scientific Papers in G7 and BRICs}

The qualitative status of SCI papers in the major research countries (G7, BRICs) was investigated using the Relatively Rank-normalized Impact Factor $\left(R^{2} n I F\right)$ from 2007 to 2009. As shown in Table 4, the US produced the SCI papers of highest quality $\left(R^{2} n I F=1.088\right)$. In 2009 the UK, France, Germany, Italy, and Canada had the $R^{2} n I F$ values of 1.038, 1.074, 1.049, 1.028, and 1.039 respectively, higher than the global average of 1.0. In particular, $R^{2} n I F$ of SCI papers in Germany increases noticeably during the same period. Although Japan is the only G7 country whose $R^{2} n I F$ is less than the global average the quality of its SCI papers was on the rise while those of France, Italy, and Canada did not change significantly during the period.

Table 3

Examples of $R^{2}$ nIF Calculation of SCI Papers

\begin{tabular}{|c|c|c|c|c|c|c|}
\hline standard & Journal & Scientific paper & SCI IF & $\begin{array}{l}m r n I F \\
(\mathrm{~A})\end{array}$ & $\begin{array}{l}m r n I F_{\text {global }} \\
\text { Average }(\mathrm{B})\end{array}$ & $\begin{array}{l}R^{2} n I F \\
(\mathrm{~A} / \mathrm{B})\end{array}$ \\
\hline \multirow{5}{*}{ Mathematics } & $\begin{array}{l}\text { Journal of the American } \\
\text { Statistical Association }\end{array}$ & $\begin{array}{l}\text { Time series modelling with } \\
\text { semiparametric... }\end{array}$ & 2.322 & 95.057 & \multirow{5}{*}{65.415} & 1.453 \\
\hline & $\begin{array}{l}\text { Journal of Differential } \\
\text { Equations }\end{array}$ & $\begin{array}{l}\text { Interior regularity criteria for suitable } \\
\text { weak solutions... }\end{array}$ & 1.426 & 79.658 & & 1.218 \\
\hline & $\begin{array}{l}\text { Journal fur Die Reine } \\
\text { und Angewandte } \\
\text { Mathematik }\end{array}$ & $\begin{array}{l}\text { Unobstructedness of deformations of } \\
\text { holomorphic maps onto... }\end{array}$ & 1.079 & 66.540 & & 1.017 \\
\hline & $\begin{array}{l}\text { European Journal of } \\
\text { Combinatorics }\end{array}$ & $\begin{array}{l}\text { On imprimitive multiplicity-free } \\
\text { permutation groups... }\end{array}$ & 0.822 & 51.141 & & 0.782 \\
\hline & Journal of Graph Theory & $\begin{array}{l}\text { Circle graph obstructions under } \\
\text { pivoting... }\end{array}$ & 0.662 & 34.791 & & 0.532 \\
\hline \multirow{5}{*}{$\begin{array}{l}\text { Molecular } \\
\text { Biology } \\
\text { \& Genetics }\end{array}$} & Genes \& development & $\begin{array}{l}\text { Bacterial-modulated host immunity and } \\
\text { stem cell... }\end{array}$ & 12.075 & 95.817 & \multirow{5}{*}{63.529} & 1.508 \\
\hline & Human Genetics & $\begin{array}{l}\text { Evidence that TGFA influences risk to } \\
\text { cleft lip... }\end{array}$ & 4.523 & 79.658 & & 1.254 \\
\hline & $\begin{array}{l}\text { European journal of cell } \\
\text { biology }\end{array}$ & $\begin{array}{l}\text { A critical role for AKT activation in } \\
\text { protecting cells... }\end{array}$ & 3.314 & 63.498 & & 1.000 \\
\hline & BMC cell biology & $\begin{array}{l}\text { Heat shock protein } 70 \text {-mediated } \\
\text { sensitization of cells... }\end{array}$ & 2.654 & 49.049 & & 0.772 \\
\hline & $\begin{array}{l}\text { Annals of Human } \\
\text { Genetics }\end{array}$ & $\begin{array}{l}\text { Association of a polymorphism in the } \\
\text { intron } 7 \text { of the SREBF1 gene... }\end{array}$ & 2.145 & 34.221 & & 0.539 \\
\hline
\end{tabular}

BRIC countries except Russia experienced a fast growth in the number of SCI papers as shown in Table 4. The growth rate of SCI papers in Brazil, China, and India is $28.3 \%, 19.0 \%$, and $16.4 \%$, respectively. However, $R^{2} n I F$ values of those papers were below the global average of 1.0. Furthermore, their $R^{2} n I F$ values show a slight decline: the $R^{2} n I F$ growth rate is $-0.20 \%$ in India, $-0.10 \%$ in Brazil, and $-0.04 \%$ in China. These results indicate that the qualitative status of scientific papers in BRIC countries is not up to par yet, although they have certainly accomplished a quantitative expansion of papers.

Table 5 shows the $R^{2} n I F$ in each NSI standard field of SCI papers produced by G7 and BRIC countries. The US produced the top quality papers in almost all science areas with the highest $R^{2} n I F$ values as shown in Figure 1. The US particularly turned out to be the research leader in both "basic science" and "bio-science". For instance, the $R^{2} n I F$ of scientific papers produced by the US is 1.123 in "chemistry", 1.111 in "physics", 1.079 in "space science", 1.141 in "immunology", 1.100 in "biology \& biochemistry", 1.086 in "clinical medicine”, and 1.089 in "multidisciplinary". The UK also had higher $R^{2} n I F$ values than the global average in almost all areas. In particular, the UK boasts the best qualities in the areas of "pharmacology \& toxicology" (1.133), “molecular biology \& genetics” (1.120), “agricultural science” (1.095), “plant \& animal science” (1.091), 
“neuroscience \& behavior” (1.082), and “environment/ecology” (1.063), with $R^{2} n I F$ values superior even to the US. For Japan and China, the $R^{2} n I F$ values were not up to par in many areas. As shown in Figure 1, Japan produced scientific papers of quality above the world average of 1.0 in only the basic science categories such as "chemistry”, "geoscience”, "physics”, "space science”, and "multidisciplinary”. China had reached the world average in eight areas, particularly "agricultural science”, "engineering”, "environment/ecology”, "geoscience”, and "materials science". Notably, in "engineering" China was the second country in journal quality after the US.

Table 4

Number and $R^{2} n I F$ of SCI Papers in G7 and BRIC Countries

\begin{tabular}{|c|c|c|c|c|c|c|c|c|c|}
\hline Country & & 2007 & 2008 & 2009 & Growth rate & 2007 & 2008 & 2009 & Growth rate \\
\hline \multirow{7}{*}{ G7 } & US & 305,922 & 340,493 & 341,038 & $5.6 \%$ & 1.088 & 1.088 & 1.088 & $0.01 \%$ \\
\hline & UK & 82,995 & 91,226 & 92,628 & $5.6 \%$ & 1.068 & 1.070 & 1.074 & $0.29 \%$ \\
\hline & France & 54,381 & 64,515 & 65,301 & $9.6 \%$ & 1.047 & 1.046 & 1.049 & $0.10 \%$ \\
\hline & Germany & 76,544 & 87,433 & 89,545 & $8.2 \%$ & 1.028 & 1.035 & 1.038 & $0.49 \%$ \\
\hline & Italy & 43,768 & 50,367 & 51,606 & $8.6 \%$ & 1.030 & 1.031 & 1.028 & $-0.10 \%$ \\
\hline & Canada & 47,230 & 53,286 & 55,534 & $8.4 \%$ & 1.038 & 1.042 & 1.039 & $0.08 \%$ \\
\hline & Japan & 73,756 & 79,515 & 78,930 & $3.4 \%$ & 0.963 & 0.966 & 0.971 & $0.43 \%$ \\
\hline \multirow{4}{*}{ BRICs } & Brazil & 19,510 & 30,422 & 32,100 & $28.3 \%$ & 0.929 & 0.932 & 0.927 & $-0.10 \%$ \\
\hline & Russia & 25,892 & 27,918 & 30,178 & $8.0 \%$ & 0.580 & 0.605 & 0.594 & $1.27 \%$ \\
\hline & India & 29,717 & 38,697 & 40,250 & $16.4 \%$ & 0.910 & 0.903 & 0.906 & $-0.20 \%$ \\
\hline & China & 90,160 & 112,835 & 127,653 & $19.0 \%$ & 0.943 & 0.938 & 0.942 & $-0.04 \%$ \\
\hline
\end{tabular}

Table 5

$R^{2} n I F$ of Main Countries (G7, BRICs) in Scientific Areas (2007-2009)

\begin{tabular}{|c|c|c|c|c|c|c|c|c|c|c|c|}
\hline \multirow{2}{*}{ NSI Standard Field } & \multicolumn{7}{|c|}{ G7 } & \multicolumn{4}{|c|}{ BRICs } \\
\hline & US & UK & France & Germany & Italy & Canada & Japan & Brazil & Russia & India & China \\
\hline Agricultural Sciences & 1.012 & 1.095 & 1.081 & 0.966 & 1.052 & 0.973 & 0.889 & 1.009 & 0.986 & 0.990 & 1.006 \\
\hline Biology \& Biochemistry & 1.100 & 1.079 & 1.043 & 1.056 & 1.004 & 1.038 & 0.936 & 0.749 & 0.752 & 0.799 & 0.855 \\
\hline Chemistry & 1.123 & 1.122 & 1.082 & 1.073 & 1.079 & 1.093 & 1.015 & 0.953 & 0.498 & 0.888 & 0.958 \\
\hline Clinical Medicine & 1.086 & 1.045 & 1.015 & 0.964 & 1.019 & 1.054 & 0.953 & 0.874 & 0.461 & 0.830 & 0.900 \\
\hline Computer Science & 1.112 & 1.027 & 0.998 & 1.035 & 1.006 & 1.057 & 0.742 & 1.018 & 0.953 & 1.000 & 0.997 \\
\hline Engineering & 1.037 & 1.007 & 1.024 & 1.029 & 1.012 & 1.000 & 0.979 & 1.025 & 0.633 & 1.006 & 1.029 \\
\hline Environment/Ecology & 1.014 & 1.063 & 1.053 & 1.034 & 0.997 & 1.003 & 0.976 & 0.953 & 0.916 & 0.960 & 1.022 \\
\hline Geosciences & 1.056 & 1.048 & 1.027 & 1.041 & 1.013 & 0.996 & 1.028 & 1.008 & 0.447 & 1.019 & 1.017 \\
\hline Immunology & 1.141 & 1.093 & 1.073 & 1.045 & 0.927 & 1.058 & 0.931 & 0.778 & 0.904 & 0.670 & 0.831 \\
\hline Materials Science & 1.061 & 1.034 & 1.026 & 1.025 & 1.016 & 0.992 & 0.933 & 0.986 & 0.793 & 0.981 & 1.016 \\
\hline Mathematics & 1.061 & 1.040 & 1.010 & 1.056 & 1.040 & 0.974 & 0.928 & 1.074 & 0.647 & 0.989 & 0.984 \\
\hline Microbiology & 1.035 & 1.018 & 1.018 & 1.008 & 0.997 & 1.016 & 0.980 & 0.944 & 0.891 & 0.951 & 0.958 \\
\hline Molecular Biology \& Genetics & 1.109 & 1.120 & 1.066 & 1.072 & 0.977 & 1.038 & 0.905 & 0.739 & 0.712 & 0.714 & 0.809 \\
\hline Multidisciplinary & 1.089 & 1.055 & 1.072 & 1.077 & 1.053 & 1.072 & 1.065 & 1.030 & 0.987 & 0.673 & 0.773 \\
\hline Neuroscience \& Behavior & 1.068 & 1.082 & 0.988 & 0.978 & 0.963 & 1.004 & 0.923 & 0.880 & 0.908 & 0.783 & 0.886 \\
\hline Pharmacology \& Toxicology & 1.101 & 1.133 & 1.093 & 1.050 & 1.093 & 1.051 & 0.869 & 0.896 & 0.989 & 0.840 & 0.844 \\
\hline Physics & 1.111 & 1.089 & 1.053 & 1.095 & 1.047 & 1.088 & 1.001 & 1.004 & 0.722 & 0.940 & 0.916 \\
\hline Plant \& Animal Science & 1.033 & 1.091 & 1.108 & 1.031 & 1.036 & 1.006 & 0.932 & 0.956 & 0.584 & 0.931 & 1.007 \\
\hline Psychiatry/Psychology & 1.028 & 1.060 & 1.018 & 0.879 & 1.028 & 0.982 & 0.977 & 0.977 & 0.946 & 0.975 & 1.027 \\
\hline Social Sciences, general & 1.031 & 0.999 & 0.998 & 0.981 & 0.952 & 0.985 & 1.002 & 1.012 & 0.681 & 0.900 & 1.024 \\
\hline Space Science & 1.079 & 1.031 & 0.966 & 1.010 & 1.003 & 1.051 & 1.050 & 0.962 & 0.751 & 0.894 & 0.935 \\
\hline
\end{tabular}



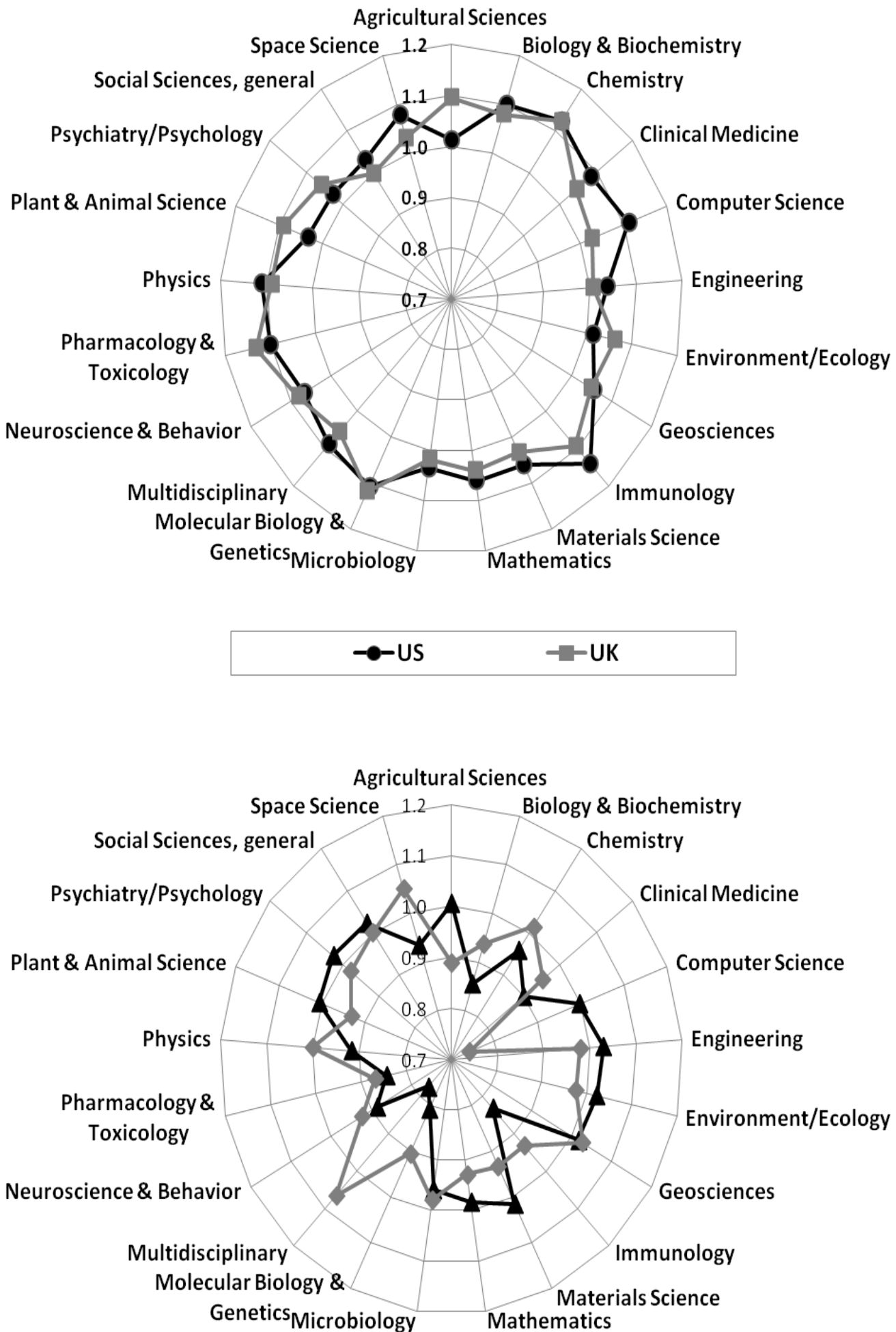

$\leftarrow$ China $\quad \rightarrow$ Japan

Figure 1. $R^{2} n I F$ of US, UK, Japan, and China in scientific areas (2007-2009). 


\section{Conclusions}

It is well known that uninformed scholars and science administrators often use the Journal Impact Factor (IF) without taking into account the variations in citation characteristics inherent in individual science areas. Scholars such as Sen (1992), Marshakova-Shaikevich (1996), and Pudovkin and Garfield (2004) attempted to develop better research performance measures ( $S n I F, M n I F, r n I F$, etc.) to overcome the limitations of Journal IF analyses, but these indicators still fail to consider the intrinsic differences in characteristics between research areas. We are suffering from the shortcomings when comparing the qualities of scientific papers produced by different countries and research areas.

This article introduces a new qualitative measurement indicator, the Relatively Rank-normalized Impact Factor $\left(R^{2} n I F\right)$, which enables us to evaluate the qualities of scientific papers with global standards. We believe this innovative indicator provides reliable and easily interpretable qualitative values of scientific publications.

With the help of $R^{2} n I F$ indicator, we study the qualitative status of SCI papers of G7 and BRIC countries in research areas delimited by the NSI standard fields. The most interesting results are as follows: Firstly, the US scored best with top quality papers in almost all scientific areas, particularly "basic science" and

"bio-science"; Secondly, the UK also showed higher qualities in almost all areas, even being superior to US in the some research areas such as "pharmacology \& toxicology", "molecular biology \& genetics", and "neuroscience \& behavior"; Thirdly, the qualitative status of all BRIC countries were not up to par in most research areas although they did achieve a quantitative growth of scientific papers during the time period of the study. Surprisingly in "engineering” China was the second in journal quality only to the US. Lastly, Japan only produced scientific papers matching the average qualitative global standard in "basic science" although its $R^{2} n I F$ was increasing.

This article provides insight into how we should utilize scientific publication as the basic criterion when evaluating the research performance of individual scientists, institutions, and R\&D programs. In the evaluation process more weight should be put on the quality rather than quantity of publications, thereby encouraging research quality through $R \& D$ funding. We believe our article serves as a useful starting point for such a discussion.

\section{References}

Gang, D., \& Moon, S. (2009). Research on bibliometric indicator information analysis for research quality evaluation. Korea Association of Information Management, 26(3), 377-394.

Garfield, E. (2001). Interview with Eugene Garfield, Chairman Emeritus of the Institute for Scientific Information (ISI). Cortex, 37(4), 575-577.

Garg, K. C., \& Padhi, P. (1999). Scientometrics of laser literature as viewed through the journal of current laser abstracts. Scientometrics, 45, 251-268.

Heo, J., Kim, H., Cho, Y., \& Cho, S. (2008). Research on development of new bibliometric indicators for scientific performance analysis for national R\&D project. Journal of Korea Technology Innovation Society, 11(3), 376-399.

Kim, Y., Yeo, M., \& Oh, D. (2010). Measuring the quality of research performance by relative rank-normalized impact factor. Asian Research Policy, 1(1), 27-42.

Kostoff, R. N. (1995). Federal research impact assessment: Axioms, approaches, applications. Scientometric, 34, $163-206$.

Leeuwen, T. N., Van Wurff, L. J., \& Raan, A. F. J. (2001). The use of combined bibliometric methods in research funding policy. Research Evaluation, 10(3), 195-201.

Marshakova-Shaikevich, I. (1996). The standard impact factor as an evaluation tool of science and scientific journals. Scientometric, 25, 283-290. 
Marshakova-Shaikevich, I. (2003). Bibliometric evaluation of Russian science journals. The Herald of Russian Academy of Sciences, 73(9), 788-796.

Norris, M., \& Oppenheim, C. (2003). Citation counts and the research assessment exercise V-Archeology and the 2001 RAE. Journal of Documentation, 59, 709-739.

Oppenheim, C. (1997). The correlation between citation counts and the 1992 research assessment exercise ratings for British research in genetics, anatomy and archaelogy. Journal of Documentation, 53, 477-487.

Pudovkin, A. I., \& Garfield, E. (2004). Rank-normalized impact factor: A way to compare journal performance across subject categories. Proceedings of the 67th ASIS\&T Annual Meeting, 41, 507-515.

Seglen, P. O. (1997). Why the impact of journals should not be used for evaluating research. British Medical Journal, 314(7079), 498-502.

Sen, B. K. (1992). Normalized impact factor. Journal of Documentation, 48, 318-329. 